\title{
Which battery model to use?
}

\author{
Marijn R. Jongerden and Boudewijn R. Haverkort \\ University of Twente \\ Faculty for EEMCS, Centre for Telematics and Information Technology \\ 7500 AE Enschede, The Netherlands \\ [jongerdenmr, brh]@ewi.utwente.nl \\ Phone/Fax: +31 53489 4095/4524 \\ http://dacs.ewi.utwente.nl/
}

\begin{abstract}
The use of mobile devices like cell phones, navigation systems, or laptop computers, is limited by the lifetime of the included batteries. This lifetime depends naturally on the rate at which energy is consumed, however, it also depends on the usage pattern of the battery. Continuous drawing of a high current results in an excessive drop of residual capacity. However, during intervals with no or very small currents, batteries do recover to a certain extent. The usage pattern of a device can be well modeled with stochastic workload models. However, one still needs a battery model to describe the effects of the power consumption on the state of the battery. Over the years many different types of battery models have been developed for different application areas. In this paper we give a detailed analysis of two well-known analytical models, the kinetic battery model and the so-called diffusion model. We show that the kinetic battery model is actually an approximation of the more complex diffusion model; this was not known previously. Furthermore, we tested the suitability of these models for performance evaluation purposes, and found that both models are well suited for doing battery lifetime predictions. However, one should not draw conclusions on what is the best usage pattern based on only a few workload traces.
\end{abstract}

\section{Introduction}

With the proliferation of cheap wireless access technologies, such as wireless LAN, Bluetooth as well as GSM, the number of wireless devices every citizen is using has been steadily increasing over the last decade. Such devices do not only add to the flexibility with which we can do our work, but also add to our reachability and our security. Next to these personal wireless devices, an ever growing number of wireless devices is used for surveillance purposes, most notably in wireless sensor networks. A common issue to be dealt with in the design of all of these devices is power consumption. Since all of these devices use batteries of some sort, mostly rechargeable, achieving low power consumption for wireless devices has become a key design issue. This fact is witnessed by many recent publications on this topic (see also [1]).

Low-power design is a very broad area in itself, with so-called "battery-driven system design" a special branch of it, that becomes, due to the reasons above, more and more important. A key issue to be addressed is to find the right tradeoff between battery usage and required performance: how can we design a (wireless) system such that with a given battery, 
good performance (throughput, reachability, and so on) is obtained, for a long-enough period. Stated differently, how should the processes in the wireless device be organised such that the battery lifetime (which determines the system lifetime) will be as high as possible. Indeed, it has been observed recently that due to the specific physical nature of batteries, achieving the longest battery lifetime is not always achieved by "just" trying to minimise the power consumption at any point in time. Instead, also the way in which the power is consumed, that is, the current-extraction patterns and the employed current levels play a role in the battery lifetime.

Using an abstract workload model one can model the operation of a system, describing the various states the wireless device can be in, together with the energy consumption rates in those states. Also, the transition possibilities between these states can be represented in the workload model. Such a description can be interpreted as a Markov-reward model in which accumulated reward stands for the amount of energy consumed. The system or battery lifetime then equals the time until a certain level of consumption (the available charge of the battery) is reached. Determining this time, or better, its distribution, can be done with wellknown techniques for performability evaluation [2]. However, such an approach does not well take into account the physical aspect of battery operation. Indeed, studies on batteries reveal that the battery depletion rate in general is non-linear in time, and, moreover, also depends on the amount of energy still in the battery $[2,3]$. Furthermore, in periods when a battery is not used, subtle but important battery-restoration effects are in place, that apparently refill the battery.

To capture the influence of the power consumption on the battery, a battery model is needed. Over the years, many different types of battery models have been developed for different application areas [3]. For example, the electro-chemical models described in $[4,5,6]$ are used in battery design. These models describe the battery in a very detailed manner using a set of six coupled differential equations. Another example are the electrical circuit models used in electrical enginering [7], which focus on the electrical properties of the battery. Although these models describe the battery accurately, they are not suitable to be used in the setting of the performance models because of the detailed description, which would make the combined model unmanageable. What is needed, is an abstract model which focuses on the important battery properties and their effects only. Two analytical models are good candidates: the Kinetic Battery Model (KiBaM) by Manwell and McGowan [8, 9, 10] and the diffusion based model by Rakhmatov and Vrudhula [11]. These two models describe the battery using only two differential equations. Although the equations the two models start from are really different, we show that the KiBaM is actually a first order approximation of the diffusion model. A further theoretical and practical comparison of the two models is made, which leads to the conclusion which model is best to use in the setting of the performance models.

The rest of the paper is structured as follows. Section 2 gives a short introduction to battery properties that have to be addressed, together with a short overview of different battery models available in the literature. In Section 3 the two analytical battery models, the Kinetic Battery Model and the Diffusion model, are described in detail and Section 4 gives a theoretical and practical comparison of these models, which leads to the conclusion which model is best to use. In Section 5 the limitations of the analytical model are discussed. We end with conclusions and an outlook to future work in Section 6. 


\section{Battery models}

In this section we give a short introduction to the essential battery properties which need to be modeled to obtain an accurate battery model. Furthermore, short descriptions of the electrochemical, electrical circuit and stochastic battery models are given. Although these models give a good description of the battery, they are not that well suited to be used for performance modelling. The two analytical models described in Section 3 are better suited for this purpose. These models are described in more detail.

\subsection{Introduction}

The two most important properties of a battery are its voltage (expressed in volts, $V$ ) and its capacity (mostly expressed in Ampere-hour, $A h$ ); the product of these two quantities is a measure for the energy stored in the battery. For an ideal battery the voltage stays constant over time until the moment it is completely discharged, then the voltage drops to zero. The capacity in the ideal case is the same for every load for the battery. Reality is different, though: the voltage drops during discharge and the effectively perceived capacity is lower under a higher load. This phenomenon is termed the rate capacity effect.

In the ideal case it is easy to calculate the lifetime of a battery. The lifetime $(L)$ in the case of a constant load is the capacity $(C)$ over the load current $(I)$ :

$$
L=C / I \text {. }
$$

Due to various nonlinear effects this relation does not hold for real batteries. A simple approximation for the lifetime under constant load can be made with Peukert's law [11]:

$$
L=\frac{a}{I^{b}}
$$

where $a>0$ and $b>1$ are constants which depend on the battery. For variable loads $(i(t))$ one can extend 2 by using the average current up until $t=L$ :

$$
L=\frac{a}{\left(\frac{1}{L} \int_{0}^{L} i(t) d t\right)^{b}}
$$

Following (3), all load profiles with the same average current have the same lifetime. Experimentally it can be shown that this is not the case. One of the effects playing an important role here is the recovery effect of the battery, i.e., the effect that the battery regains some of its "lost" capacity during idle periods.

\subsection{Electrochemical models}

The electrochemical models are based on the chemical processes that take place in the battery. The models describe these battery processes in great detail. This makes these models the most accurate battery models. However, the highly detailed description makes the models complex and difficult to configure.

Doyle, Fuller and Newman developed an electrochemical model for lithium and lithiumion cells $[4,5,6]$. This model consists of six coupled, non-linear differential equations. Solving these equations gives the voltage and current as functions of time, as well as the potentials 
in the electrolyte and electrode phases, salt concentration, reaction rate and current density in the electrolyte as functions of time and position in the cell.

Dualfoil is a Fortran program that uses this model to simulate lithium-ion batteries. The program is freely available on the internet [12]. It computes how all the battery properties change over time for the load profile set by the user. From the output data, it is possible to obtain the battery lifetime. Besides the load profile, the user has to set over 50 battery related parameters, e.g., the thickness of the electrodes, the initial salt concentration in the electrolyte and the overall heat capacity. To be able to set all these parameters one needs a very detailed knowledge of the battery that is to be modelled. On the other hand, the accuracy of the program is very high. The program is often used as a comparison against other models, instead of using experimental results to check the accuracy, as we do in Section 4.3 .

\subsection{Electrical-circuit models}

In electrical-circuit models the electrical properties of the battery are modeled using PSpice circuits consisting of voltage sources, lookup tables and linear passive elements, such as resistors and capacitors. The first electrical-circuit models were proposed by Hageman [7]. He used simple PSpice circuits to simulate nickel-cadmium, lead-acid and alkaline batteries. Gold [13] proposed a similar model for lithium-ion batteries. The core of the models for the different types of batteries is the same:

- a capacitor represents the capacity of the battery,

- a discharge-rate normaliser determines the lost capacity at high discharge currents,

- a circuit to discharge the capacity of the battery,

- a voltage versus state-of-charge lookup table,

- a resistor representing the battery's resistance.

Figure 1 shows the basic circuits used to model an arbitrary cell. Minor changes have to be made to complete the model for a specific cell type. Although the models are simpler than the electrochemical models and therefore computationally less expensive, it still takes quite some effort to configure them. Especially the lookup tables used in the model require much experimental data on the battery's behaviour. Furthermore, the models are less accurate in predicting battery lifetime, having errors up to approximately $12 \%$ [13].

\subsection{Stochastic models}

The stochastic model developed by Chiasserini and Rao describes the battery at a higher level of abstraction than the electrochemical and electrical circuit models. Between 1999 and 2001 Chiasserini and Rao published a series of papers on battery modelling based on discrete-time Markov chains $[14,15,16,17]$. In [14] two models of a battery of a mobile communication device for transmitting packets are described. In the first and simplest model, the battery is described by a discrete time Markov chain with $N+1$ states, numbered from 0 to $N$ (cf. Figure 2 ). The state number corresponds to the number of charge units available in the battery. One charge unit corresponds to the amount of energy required to transmit a single packet. $N$ is the number of charge units directly available based on continuous use. In this simple model, 


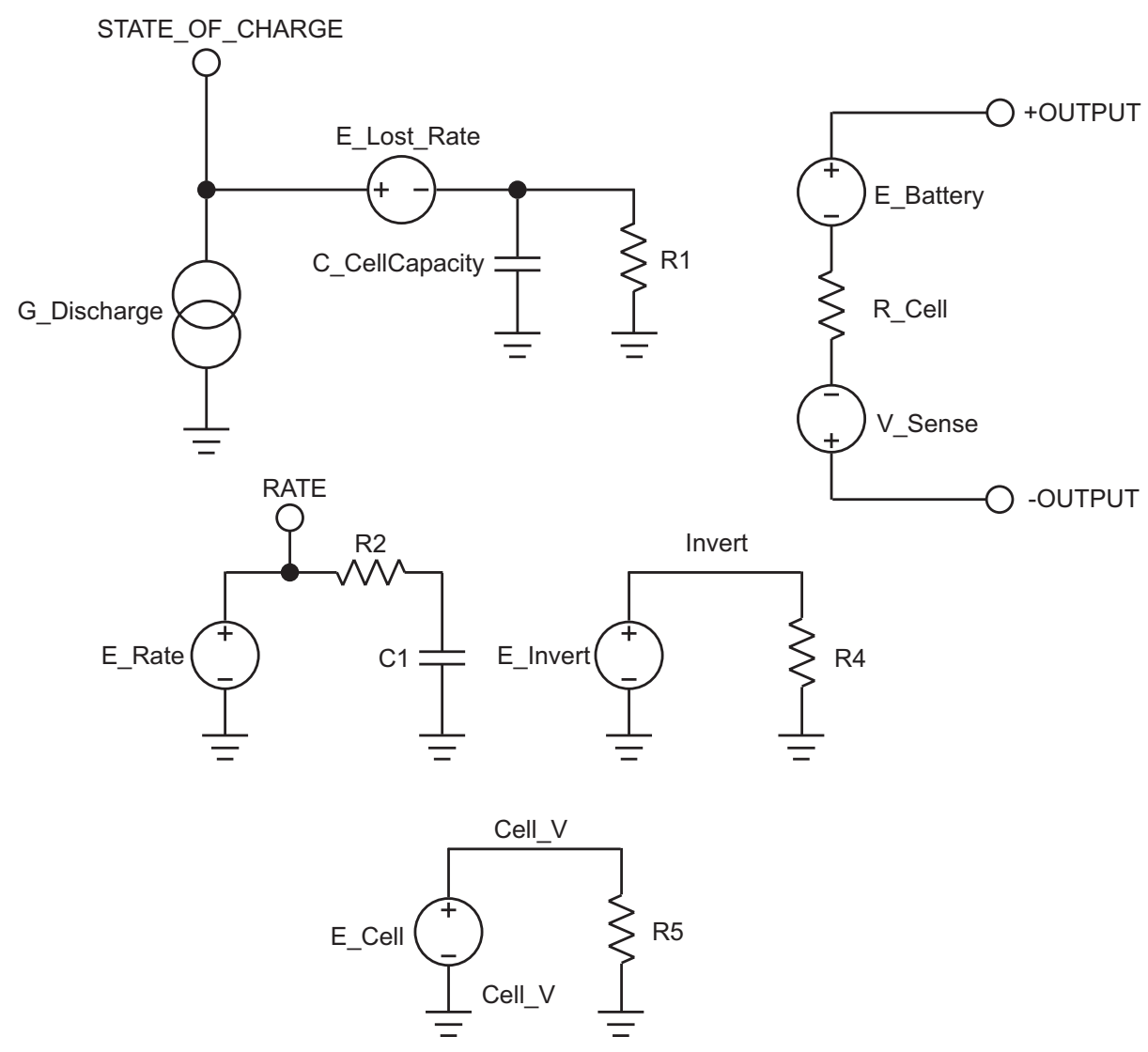

Figure 1: Basic functional schematic covering all the modeled cell types. This basic schematic requires minor changes to complete the models for each specific cell [7].

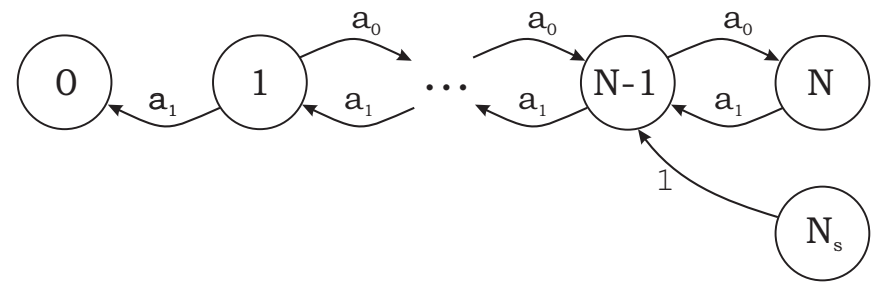

Figure 2: The basic Markov chain battery model by Chiasserini and Rao [14] 


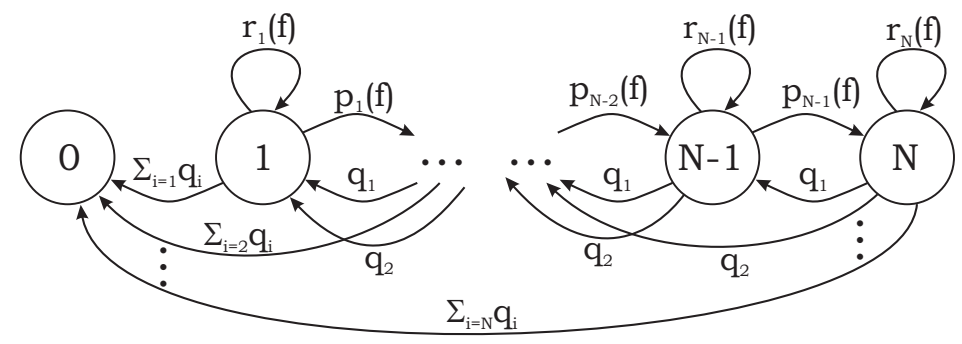

Figure 3: The extended Markov chain battery model by Chiasserini and Rao [17]

every time step either a charge unit is consumed with probability $a_{1}=q$ or recovery of one unit of charge takes place with probability $a_{0}=1-q$. The battery is considered empty when the absorbing state 0 is reached or when a maximum of $T$ charge units have been consumed. The number of $T$ charge units is equal to the theoretical capacity of the battery $(T>N)$.

For this simple model it is possible to give analytical expressions for the properties of interest. The main properties investigated are the expected number of transmitted packets $\left(\bar{m}_{p}\right)$ and the gain $(G)$ obtained from a pulsed discharge relative to a constant discharge, defined as: $G=\bar{m}_{p} / N$. Clearly, pulsed discharge will lead to a gain that exceeds 1, due to the possibility to recover some charge units. However, this model is too simple. The rate of recovery is not constant during discharge, and in most systems the discharge current changes over time.

In the models in $[15,16,17]$ several extensions are made to solve these problems. To improve the model, the recovery probability is made state dependent. When less charge units are available the probability to recover a charge unit will become smaller. Next to the state dependence of the recovery, there is a phase dependence. The phase number $(f)$ is a function of the number of charge units that has been consumed. When more charge units have been consumed, the phase number increases and this causes the probability of recovery to decrease. Also, it is possible to consume more than one charge unit in any one time step, with a maximum of $M$ charge units $(M \leq N)$. In this way a more bursty consumption of energy can be modeled. Another aspect that has been added to the model, is the non-zero probability of staying in the same state. This means no energy consumption or recovery takes place during a time step.

In Figure 3, the state transition diagram of the model with all the extensions is shown. With probability $q_{i}, i$ charge units are requested in one time slot. During the idle periods, the battery either recovers one charge unit with probability $p_{j}(f)$, or stays in the same state with probability $r_{j}(f)$. The recovery probability in state $j$ and phase $f$ is defined as [17]:

$$
p_{j}(f)=q_{0} e^{(N-j) g_{N}-g_{C}(f)},
$$

where $g_{N}$ and $g_{C}(f)$ depend on the recovery behaviour of the battery. One can model different loads by setting the transition probabilities appropriately.

In [17] the final version of the model is used to model a lithium-ion battery. To model the battery, $N$ is set to $\sim 2 \times 10^{6}$, and 2 phases are used. This results in a Markov chain with approximately $4 \times 10^{6}$ states. The model is analysed by numerical computations, and the results are compared with Dualfoil (cf. Section 2.2) [17]. With both models, the gain obtained from pulsed discharge compared to constant discharge is calculated for different 


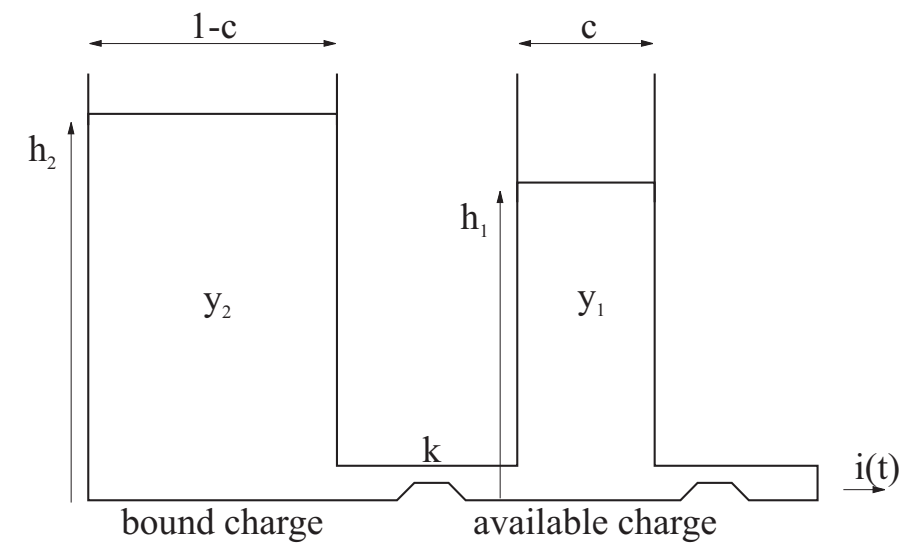

Figure 4: Two-well-model of the Kinetic Battery Model

discharge currents. The results of the stochastic model have a maximum deviation of $4 \%$ from the electro-chemical model, with an average deviation of $1 \%$. These results show that the stochastic model gives a good qualitative description of battery behaviour under pulsed discharge. However, it is unclear how well the model performs quantitatively, since only results of the gain and no numbers for the computed lifetimes are given.

\section{Analytical models}

In this section a detailed description of two analytical battery models is given. Both models describe the non-linear effects of the battery using two differential equations. In Section 3.1 we present the Kinetic Battery Model of Manwell and McGowan. To get more insight in this model, we apply a coordinate transformation in Section 3.2. In Section 3.3 we present the diffusion model of Rakhmatov and Vrudhula. Then, in Section 3.4, we show that the diffusion model is the continuous version of the Kinetic Battery Model.

\subsection{Kinetic Battery Model}

The first analytical model we discuss, is the Kinetic Battery Model (KiBaM) of Manwell and McGowan [8, 9, 10]. The KiBaM is a very intuitive battery model. It is called kinetic because it uses a chemical kinetics process as its basis. In the model, the battery charge is distributed over two wells: the available-charge well and the bound-charge well (cf. Figure 4). A fraction $c$ of the total capacity is put in the available charge well (denoted $y_{1}(t)$ ), and a fraction $1-c$ in the bound charge well (denoted $y_{2}(t)$ ). The available charge well supplies electrons directly to the load $(i(t))$, whereas the bound-charge well supplies electrons only to the available-charge well. The charge flows from the bound charge well to the available charge well through a "valve" with fixed conductance, $k$. Next to this parameter, the rate at which charge flows between the wells depends on the height difference between the two wells. The heights of the two wells are given by: $h_{1}=y_{1} / c$ and $h_{2}=y_{2} / 1-c$. The change of the 
charge in both wells is given by the following system of differential equations:

$$
\left\{\begin{array}{l}
\frac{d y_{1}}{d t}=-i(t)+k\left(h_{2}-h_{1}\right) \\
\frac{d y_{2}}{d t}=-k\left(h_{2}-h_{1}\right)
\end{array}\right.
$$

with initial conditions $y_{1}(0)=c \cdot C$ and $y_{2}(0)=(1-c) \cdot C$, where $C$ is the total battery capacity. The battery is considered empty when there is no charge left in the available charge well.

When a load is applied to the battery, the available charge reduces, and the height difference between the two wells grows. When the load is removed, charge flows from the bound-charge well to the available-charge well until $h_{1}$ and $h_{2}$ are equal again. So, during an idle period, more charge becomes available and the battery lasts longer than when the load is applied continuously. In this way the recovery effect is taken into account. Also, the rate capacity effect is covered, since for a higher discharge current the available charge well will be drained faster, less time will be available for the bound charge to flow to the available charge. Therefore, more charge will remain unused, and the effective capacity is lower.

The differential equations (5) can be solved for the case of a constant discharge current $(i(t)=I)$ using Laplace transforms, which yields:

$$
\left\{\begin{array}{l}
y_{1}(t)=y_{1,0} e^{-k^{\prime} t}+\frac{\left(y_{0} k^{\prime} c-I\right)\left(1-e^{-k^{\prime} t}\right)}{k^{\prime}}-\frac{I c\left(k^{\prime} t-1+e^{-k^{\prime} t}\right)}{k^{\prime}}, \\
y_{2}(t)=y_{2,0} e^{-k^{\prime} t}+y_{0}(1-c)\left(1-e^{-k^{\prime} t}\right)-\frac{I(1-c)\left(k^{\prime} t-1+e^{-k^{\prime} t}\right)}{k^{\prime}},
\end{array}\right.
$$

where $k^{\prime}$ is defined as $k^{\prime}=k / c(1-c), y_{1,0}$ and $y_{2,0}$ are the amount of available and bound charge, respectively, at $t=0$, and $y_{0}=y_{1,0}+y_{2,0}$.

\subsection{Coordinate transformation}

Although the differential equations (5) nicely describe the discharge process of the battery, and an analytical solution can be obtained for constant discharge currents, the equations can be made more simple when a coordinate transformation is applied. In this way even more insight can be obtained in the way the model behaves.

From (5) one can see that the height difference between the two wells $\left(h_{2}-h_{1}\right)$ plays a major role in the model. This is one of the coordinates after the transformation, the other is the total charge in the battery. So, the transformation changes the coordinates from $y_{1}$ and $y_{2}$ to $\delta=h_{2}-h_{1}$ and $\gamma=y_{1}+y_{2}$. This transformation changes the differential equations to:

$$
\left\{\begin{array}{l}
\frac{d \gamma}{d t}=-i(t) \\
\frac{d \delta}{d t}=\frac{i(t)}{c}-k^{\prime} \delta
\end{array}\right.
$$

with initial conditions $\delta(0)=0$ and $\gamma(0)=C$. In the new coordinate system the condition for the battery to be empty is: $\gamma(t)=(1-c) \delta(t)$. These differential equations are independent and are straightforwardly solved for constant discharge currents:

$$
\left\{\begin{array}{l}
\gamma(t)=C-I t \\
\delta(t)=\frac{I}{c} \cdot \frac{1-e^{-k^{\prime} t}}{k^{\prime}}
\end{array}\right.
$$




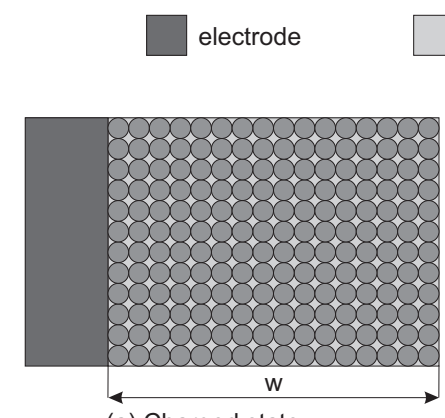

(a) Charged state

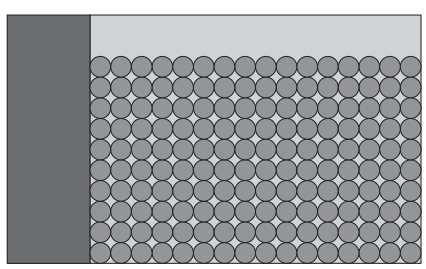

(c) After recovery electrolyte $\quad 8$ electro-active species

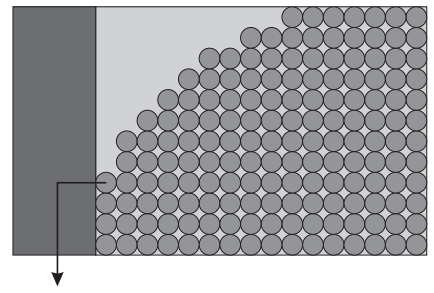

(b) Before recovery

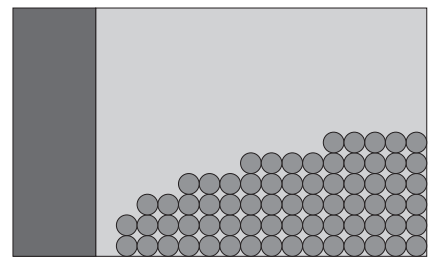

(d) Discharged state

Figure 5: Physical picture of the model by Rakhmatov and Vrudhula

During idle periods the height difference will decrease due to the flow of charge from the bound charge well to the available charge well. One can compute the function that describes the evolution of the height difference during an idle period after a load $I$ that lasted for a period of length $t_{l}$ :

$$
\delta\left(t_{i}\right)=\frac{I}{c} \cdot \frac{e^{-k^{\prime} t_{i}}\left(1-e^{-k^{\prime} t_{l}}\right)}{k^{\prime}},
$$

where $t_{i}$ is the idle time. The solutions for continuous discharge can be used to obtain a solution for any discharge profile with piecewise constant currents by adapting the initial conditions appropriately. The level of $\gamma$ and $\delta$ at the end of a step in the load profile, can then be used as initial conditions for the next step.

\subsection{Rakhmatov and Vrudhula's diffusion model}

Another analytical battery model is the one developed by Rakhmatov and Vrudhula in 2001 $[11,18,19]$. This model is based on the diffusion of the ions in the electrolyte. The model describes the evolution of the concentration of the electro-active species in the electrolyte to predict the battery lifetime under a given load. In the model the processes at both electrodes are assumed identical, thus the battery is assumed symmetric with respect to the electrodes and only one of the electrodes is considered.

Figure 5 shows a simplified view of the battery operation according to the diffusion model. At first, for the full battery, the concentration of the electro-active species is constant over the full width $(w)$ of the electrolyte (Figure 5(a)). When a load is applied to the battery, the electrochemical reaction results in a reduction of the concentration of the species near the electrode. Thus, a gradient is created across the electrolyte (Figure 5(b)). This gradient causes the species to diffuse towards the electrode. Now, when the load is switched off, 
the concentration of the species at the electrode will increase again (recover) due to the diffusion, and eventually the species will be evenly distributed over the electrolyte again. The concentration, however, will be lower than for the full battery (Figure 5(c)). Finally, when the concentration at the electrode drops below a certain value $\left(C_{\text {cutoff }}\right)$, the chemical reaction can no longer be maintained and the battery is considered to be empty (Figure 5(d)).

The concentration of the electro-active species at time $t$ and distance $x \in[0, w]$ is denoted by $C(x, t)$. For the full battery the concentration is constant over the length of the electrolyte: $C(x, 0)=C^{*}, x \in[0, w]$. The battery is considered empty when $C(0, t)$ drops below the cutoff level $C_{\text {cutoff. }}$. The evolution of the concentration is described by Fick's laws [11]:

$$
\left\{\begin{array}{l}
-J(x, t)=D \frac{\partial C(x, t)}{\partial x} \\
\frac{\partial C(x, t)}{\partial t}=D \frac{\partial^{2} C(x, t)}{\partial x^{2}}
\end{array}\right.
$$

where $J(x, t)$ is the flux of the electro-active species at time $t$ and distance $x$ from the electrode, and $D$ is the diffusion constant. The flux at the electrode surface $(x=0)$ is proportional to the current $(i(t))$. The flux on the other side of the diffusion region $(x=w)$ equals zero. This leads to the following boundary conditions:

$$
\left\{\begin{array}{l}
\left.D \frac{\partial C(x, t)}{\partial x}\right|_{x=0}=\frac{i(t)}{\nu F A} \\
\left.D \frac{\partial C(x, t)}{\partial x}\right|_{x=w}=0
\end{array}\right.
$$

where $A$ is the area of the electrode surface, $F$ is Faraday's constant, and $\nu$ is the number of electrons involved in the electrochemical reaction at the electrode surface.

It is possible to obtain an analytical solution for the set of partial differential equations (10) together with the initial condition and the boundary conditions (11) using Laplace transforms. From that solution one can obtain an expression for the apparent charge lost from the battery $(\sigma(t))[20]$ :

$$
\sigma(t)=\underbrace{\int_{0}^{t} i(\tau) d \tau}_{l(t)}+\underbrace{\int_{0}^{t} i(\tau)\left(2 \sum_{m=1}^{\infty} e^{-\beta^{2} m^{2}(t-\tau)}\right) d \tau}_{u(t)}
$$

where $\beta=\pi \sqrt{D} / w$. The apparent charge lost can be separated in two parts: the charge lost to the load $(l(t))$ and the unavailable charge $(u(t))$. The first is the charge used by the device. The second is charge which remains unused in the battery. The battery is empty when the apparent charge lost is equal to the battery's capacity.

For a constant current $I,(12)$ can easily be solved. For $l(t)$ one obtains: $l(t)=I t$. For the unavailable charge one can interchange the integral and the summation, which leads to:

$$
u(t)=2 I \sum_{m=1}^{\infty} \frac{1-e^{-\beta^{2} m^{2} t}}{\beta^{2} m^{2}} .
$$

During idle periods the unavailable charge will decrease and will be available again for the load. One can compute the function that describes the evolution of the unavailable charge 
during an idle period after a load $I$ that lasted for a period of length $t_{l}$ :

$$
u\left(t_{i}\right)=2 I \sum_{m=1}^{\infty} \frac{e^{-\beta^{2} m^{2} t_{i}}\left(1-e^{-\beta^{2} m^{2} t_{l}}\right)}{\beta^{2} m^{2}},
$$

where $t_{i}$ is the idle time.

\subsection{Continuous KiBaM}

When one compares the two analytical models, one sees many similarities. In both models the charge in the battery has to flow "to one side" to be available for usage, and part of it will stay behind in the battery when the battery is empty. One can show that the diffusion model actually is a continuous version of the KiBaM. To do this, one first needs to normalise the width $w$ (cf. Figure 5 ) of the battery, $x^{\prime}=x / w$. Now, $x^{\prime}$ is a dimensionless space coordinate, and takes a value between 0 and 1 . This changes the differential equations (10) into:

$$
\left\{\begin{array}{l}
-J\left(x^{\prime}, t\right)=\frac{D}{w} \frac{\partial C\left(x^{\prime}, t\right)}{\partial x^{\prime}} \\
\frac{\partial C\left(x^{\prime}, t\right)}{\partial t}=\frac{D}{w^{2}} \frac{\partial^{2} C\left(x^{\prime}, t\right)}{\partial x^{\prime 2}}
\end{array}\right.
$$

and the boundary conditions into:

$$
\left\{\begin{array}{l}
\left.\frac{D}{w} \frac{\partial C\left(x^{\prime}, t\right)}{\partial x^{\prime}}\right|_{x^{\prime}=0}=\frac{i(t)}{\nu F A} \\
\left.\frac{D}{w} \frac{\partial C\left(x^{\prime}, t\right)}{\partial x^{\prime}}\right|_{x^{\prime}=1}=0
\end{array}\right.
$$

Next step is to transform the ion concentration $\left(C\left(x^{\prime}, t\right)\right.$ in $\left.\mathrm{mol} / \mathrm{m}^{2}\right)$ into charge $\left(h\left(x^{\prime}, t\right)\right.$ in As). Every ion yields $\nu$ electrons in the chemical reactions, so $h\left(x^{\prime}, t\right)=C\left(x^{\prime}, t\right) A \nu F$. This yields

$$
\begin{cases}-J\left(x^{\prime}, t\right) \nu F A & =\frac{D}{w} \frac{\partial h\left(x^{\prime}, t\right)}{\partial x^{\prime}} \\ \frac{\partial h\left(x^{\prime}, t\right)}{\partial t} & =\frac{D}{w^{2}} \frac{\partial^{2} y\left(x^{\prime}, t\right)}{\partial x^{\prime 2}}\end{cases}
$$

for the differential equations and

$$
\left\{\begin{array}{l}
\left.\frac{D}{w} \frac{\partial h\left(x^{\prime}, t\right)}{\partial x^{\prime}}\right|_{x^{\prime}=0}=i(t) \\
\left.\frac{D}{w} \frac{\partial h\left(x^{\prime}, t\right)}{\partial x^{\prime}}\right|_{x^{\prime}=1}=0
\end{array}\right.
$$

for the boundary conditions. Finally, the spatial coordinate $x^{\prime}$ is discretised. Figure 6 gives a schematic overview of the discretised model. The electrolyte is divided in $n$ parts of size $\alpha=1 / n$. The level of the charge in part $i$ is denoted by $h_{i}$. We apply the finite difference method for second-order derivatives, as follows,

$$
\frac{\partial^{2} h}{\partial x^{2}}=\frac{h(x+\alpha)-2 h(x)+h(x-\alpha)}{\alpha^{2}},
$$




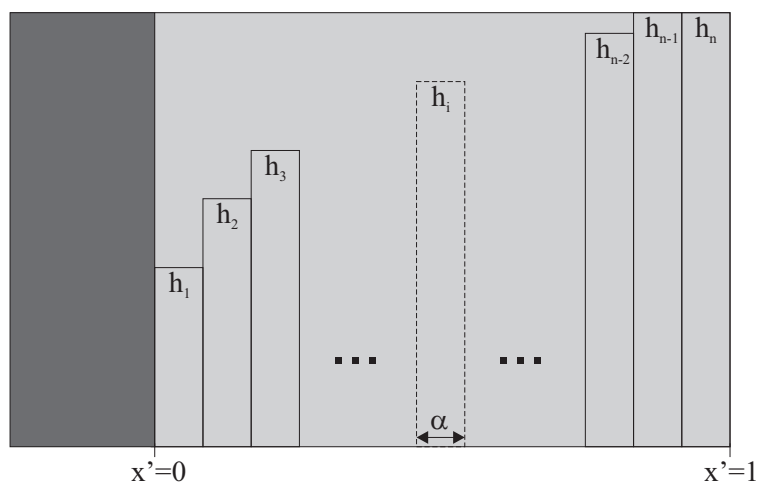

Figure 6: Discretisation of the diffusion model

where $\alpha$ is the step-size of the discretisation. For $h(0, t)$ we write $h_{1}(t)$, and for $h(1, t)$ we write $h_{n}(t)$. This turns the differential equations together with the boundary conditions into a system of $n$ coupled differential equations:

$$
\left\{\begin{aligned}
\frac{\partial h_{1}(t)}{\partial t} & =\frac{1}{\alpha}\left(\frac{D}{\alpha w^{2}}\left(h_{2}-h_{1}\right)-i(t)\right), \\
\frac{\partial h_{2}(t)}{\partial t} & =\frac{1}{\alpha}\left(\frac{D}{\alpha w^{2}}\left(\left(h_{3}-h_{2}\right)-\left(h_{2}-h_{1}\right)\right)\right), \\
& \vdots \\
\frac{\partial h_{n-1}(t)}{\partial t} & =\frac{1}{\alpha}\left(\frac{D}{\alpha w^{2}}\left(\left(h_{n}-h_{n-1}\right)-\left(h_{n-1}-h_{n-2}\right)\right)\right), \\
\frac{\partial h_{n}(t)}{\partial t} & =\frac{1}{\alpha}\left(-\frac{D}{\alpha w^{2}}\left(h_{n}-h_{n-1}\right)\right) .
\end{aligned}\right.
$$

These equations are exactly the equations one would get when the KiBaM models is extended to $n$ equally sized wells. The variable $h_{i}(t)$ gives the height of well number $i$ at time $t$. When $n=2$ the model is reduced to the KiBaM with two wells, each containing half of the total charge, that is, $c=0.5$, and $k=2 D / w^{2}$.

\section{Comparing the analytical models}

In the Section 3.4 we have shown that the diffusion model is a continuous version of the KiBaM. In this section we make a further comparison of the two models. In Section 4.1 and Section 4.2 a further theoretical comparison is made. In Section 4.3 a practical comparison is made by using both models to compute battery lifetimes for various loads.

\subsection{Continuous discharge}

It is possible to write the solution of the transformed KiBaM in the form of the diffusion model with the charge lost split in a lost to the load and an unavailable charge part. The unavailable charge in the KiBaM is the height difference times $1-c$. For constant current discharge this yields:

$$
\begin{aligned}
l(t) & =C-\gamma(t)=I t \\
u(t) & =(1-c) \cdot \delta(t)=\frac{(1-c) I}{c} \frac{1-e^{-k^{\prime} t}}{k^{\prime}}
\end{aligned}
$$




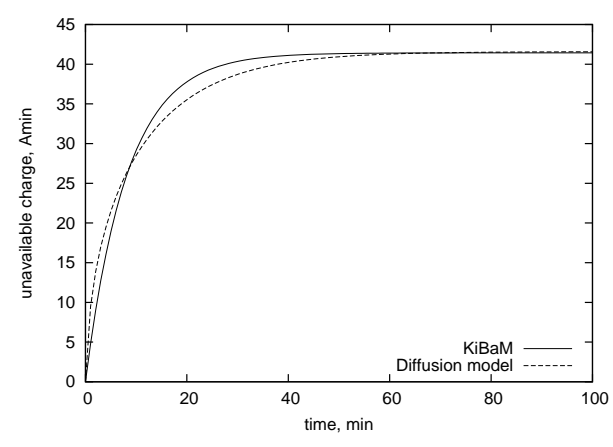

(a) Fit

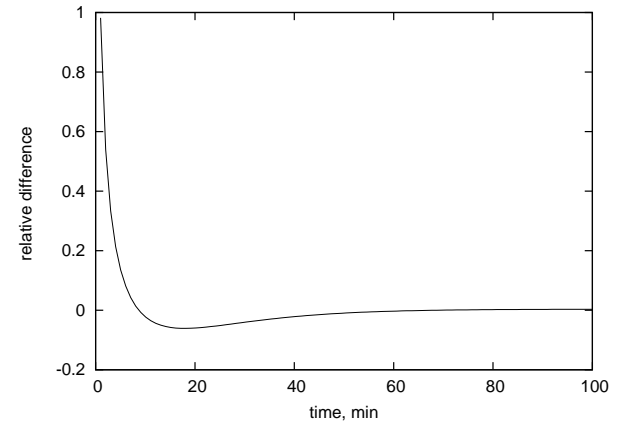

(b) relative difference

Figure 7: Fit of the KiBaM to the diffusion model. The evolution of the unavailable charge in both the diffusion model and the fitted KiBaM is given in (a). In (b) the relative difference between the two curves is given.

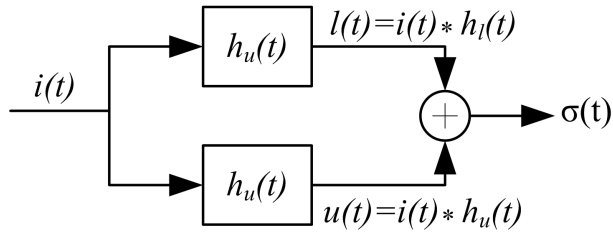

Figure 8: Linear time-invariant system of the battery model [20]

When one compares (22) with the first-order expansion of (13):

$$
u(t)=2 I \cdot \frac{1-e^{-\beta^{2} t}}{\beta^{2}},
$$

one sees the two have the same form. Setting $c=\frac{1}{3}$ and $k^{\prime}=\beta^{2}$ in the KiBaM, results in the first-order approximation of the diffusion model. This is of course, a bad approximation of the infinite sum.

One can obtain a much better approximation, when the parameters $c$ and $k^{\prime}$ are used to fit the KiBaM equation of $u(t)$ to the equation of the diffusion model. Figure 7(a) shows the result of a least squares fitting procedure for the case that $I=1 \mathrm{~A}$. When $\beta=0.273 \mathrm{~min}^{-\frac{1}{2}}$, the fit results in $c=0.166$ and $k^{\prime}=0.122 \mathrm{~min}^{-1}$. In Figure $7(\mathrm{~b})$ the relative difference between the two curves is shown. This difference is independent of the discharge current. The relative difference is very large, up to $80 \%$, for times smaller than 10 minutes. When the battery lifetime is within this region, i.e., when the battery is discharged with a high current, the results for battery lifetime computations will give a big difference.

\subsection{Frequency response}

Following the method described in [20], an analysis of the frequency response of both the Kinetic Battery Model and Rakhmatov and Vrudhula's diffusion model was done. In this method the battery model is represented by the linear time-invariant (LTI) system shown in Figure 8. For both battery models, $h_{l}(t)$ is the unit step function. For the diffusion model 


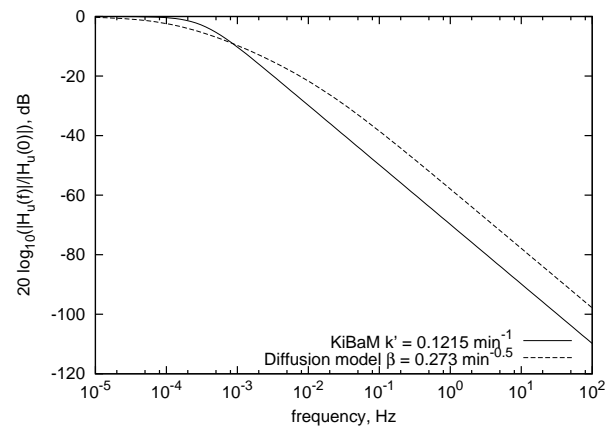

Figure 9: Frequency response for KiBaM and diffusion model

$h_{u}(t)$ is given by

$$
h_{u}(t)=2 \sum_{m=1}^{\infty} e^{-\beta^{2} m^{2} t}
$$

and for the KiBam it is

$$
h_{u}(t)=\frac{1-c}{c} e^{k^{\prime} t}
$$

For both models only $h_{u}(t)$ depends on the battery parameters. Therefore, it is sufficient to find the Fourier transform of $h_{u}(t)\left(H_{u}(f)\right)$ to characterize the frequency response of the battery. The results of the analysis are given in Figure 9.

The figure shows that the diffusion model has a higher frequency response for high frequencies. This is due to the higher-order terms that are included in the diffusion model and not in the KiBaM. However, both models are highly insensitive to high frequency current switching, in the ideal case the frequency response is zero for all frequencies. The insensitivity implies that currents varying faster than $0.01 \mathrm{~Hz}$ can be replaced with an average current without giving significant errors in the battery lifetime computations. Therefore, it is not useful to schedule tasks at small time scales, smaller than minutes, in order to benefit from the recovery effect, since the average current will stay the same. Ordering tasks at processor level will not have any effect on the battery lifetime. However, scheduling on a larger time scale, minutes or longer, can be beneficial.

The frequency response is mainly determined by the size of the recovery parameter $\left(k^{\prime}\right.$ or $\beta)$. When this parameter is increased, the recovery will be faster and the battery behaviour will be closer to that of an ideal battery. So, an increase of this parameter results in a higher frequency response, hence, to a higher sensitivity to fine-grained scheduling.

\subsection{Computing lifetimes}

Next to the theoretical analysis of the two models, both models were used to compute battery lifetimes for various load profiles.

In [19] Rakhmatov et al. give the battery lifetimes for load profiles of a Compaq Itsy pocket computer, computed both with their diffusion model and the electro-chemical model Dualfoil [12] (cf. Section 2.2. To these results, the lifetimes according to the KiBaM model have been added in Table 1 for constant loads and Table 2 for variable-load profiles. Details of the variable-load profiles are given in Table 3 (in Appendix A). 


\begin{tabular}{ccc|ccccc}
\hline Test & Name & $\begin{array}{c}I_{\text {ave }} \\
(\mathrm{mA})\end{array}$ & $\begin{array}{c}\text { Dualfoil } \\
(\mathrm{min})\end{array}$ & $\begin{array}{c}\text { Diffusion } \\
(\mathrm{min})\end{array}$ & $\begin{array}{c}\text { KiBaM } \\
(\mathrm{min})\end{array}$ & $\begin{array}{c}\text { Peukert } \\
(\mathrm{min})\end{array}$ & $\begin{array}{c}\text { Ideal } \\
(\mathrm{min})\end{array}$ \\
\hline \hline T1 & MPEG & 222.7 & 140.9 & 139.9 & 139.9 & 154.5 & 181.3 \\
T2 & Dictation & 204.5 & 156.0 & 156.0 & 156.0 & 168.4 & 197.4 \\
T3 & Talk1 & 108.3 & 317.2 & 331.4 & 331.4 & 321.3 & 372.8 \\
T4 & Talk2 & 107.5 & 319.5 & 334.1 & 334.1 & 323.7 & 375.6 \\
T5 & Talk3 & 94.9 & 365.1 & 384.0 & 384.0 & 367.5 & 425.4 \\
T6 & WAV1 & 84.3 & 413.7 & 437.5 & 437.5 & 414.4 & 478.9 \\
T7 & WAV2 & 75.5 & 464.8 & 493.3 & 493.3 & 463.6 & 534.8 \\
T8 & Idel1 & 28.0 & 1278 & 1400 & 1401 & 1270 & 1442 \\
T9 & Idle2 & 19.5 & 1852 & 2029 & 2029 & 1835 & 2071 \\
T10 & SleepDC & 3.0 & 12285 & 13417 & 13417 & 12288 & 13458 \\
T11 & IAT & 628.0 & 26 & 26.6 & 24.9 & 53.9 & 64.3 \\
T12 & IAR & 494.7 & 41.3 & 41.4 & 40.5 & 68.6 & 81.6 \\
T13 & IST & 425.6 & 54.6 & 53.9 & 53.5 & 80.0 & 94.9 \\
T14 & ISR & 292.3 & 99.5 & 96.7 & 96.7 & 117.2 & 138.1 \\
T15 & IAD & 265.6 & 113.1 & 110.6 & 110.6 & 129.1 & 152.0 \\
T16 & MSD & 252.3 & 120.8 & 118.6 & 118.6 & 136.1 & 160.0 \\
T17 & DSD & 234.1 & 132.7 & 131.0 & 131.0 & 146.8 & 172.5 \\
T18 & TSD & 137.9 & 243.6 & 251.3 & 251.3 & 251.4 & 292.8 \\
T19 & WSD & 113.9 & 300.1 & 313.0 & 313.0 & 305.3 & 354.5 \\
T20 & ISD & 57.6 & 616.3 & 659.5 & 659.5 & 610.3 & 701.0 \\
T21 & SSD & 32.5 & 1101 & 1201 & 1201 & 1092 & 1242 \\
T22 & Boot & 300.0 & 96.0 & 93.2 & 93.1 & 114.1 & 134.6 \\
\hline
\end{tabular}

Table 1: Battery lifetimes for continuous current discharge computed with Dualfoil, the diffusion model, KiBaM, and the formula's of Peukert's law and the ideal battery. The numbers for Dualfoil, the diffusion model and Peukert's law have been taken from [19]. 


\begin{tabular}{l|ccccc}
\hline Case & $\begin{array}{c}\text { Dualfoil } \\
(\min )\end{array}$ & $\begin{array}{c}\text { Diffusion } \\
(\min )\end{array}$ & $\begin{array}{c}\text { KiBaM } \\
(\min )\end{array}$ & $\begin{array}{c}\text { Peukert } \\
(\min )\end{array}$ & $\begin{array}{c}\text { Ideal } \\
(\mathrm{min})\end{array}$ \\
\hline \hline C1 & 36.4 & 36.2 & 36.3 & 60.5 & 70.8 \\
C2 & 57.2 & 55.8 & 55.7 & 79.1 & 91.9 \\
C3 & 74.2 & 71.9 & 71.4 & 93.8 & 108.5 \\
C4 & 128.1 & 124.9 & 123.6 & 142.5 & 163.0 \\
C5 & 178.5 & 176.7 & 175.7 & 190.2 & 216.5 \\
C6 & 41.5 & 41.0 & 41.1 & 64.4 & 74.7 \\
C7 & 30.6 & 30.8 & 30.5 & 56.5 & 66.9 \\
C8 & 37.0 & 37.4 & 38.1 & 60.5 & 70.8 \\
C9 & 35.4 & 35.2 & 34.8 & 60.5 & 70.8 \\
C10 & 135.2 & 132.6 & 131.7 & 148.8 & 171.3 \\
C11 & 108.8 & 107.4 & 107.9 & 148.8 & 171.3 \\
C12 & 159.0 & 155.4 & 154.1 & 174.1 & 169.3 \\
C13 & 133.8 & 131.7 & 131.3 & 148.8 & 171.3 \\
C14 & 132.9 & 129.7 & 129.4 & 148.8 & 171.3 \\
C15 & 207.6 & 209.2 & 209.2 & 216.2 & 242.1 \\
C16 & 202.4 & 200.7 & 200.7 & 216.2 & 242.1 \\
C17 & 253.8 & 251.2 & 250.8 & 266.7 & 292.1 \\
C18 & 204.6 & 204.6 & 204.3 & 216.2 & 242.1 \\
C19 & 209.4 & 208.7 & 208.2 & 221.2 & 247.1 \\
C20 & 31.7 & 33.2 & 31.5 & 60.5 & 71.9 \\
C21 & 55.9 & 55.9 & 58.8 & 85.9 & 102.5 \\
C22 & 97.5 & 94.5 & 94.3 & 117.9 & 126.6 \\
\hline & & & & & \\
\hline
\end{tabular}

Table 2: Battery lifetimes for variable-load profiles (cf. Appendix A) computed with Dualfoil, the diffusion model, KiBaM, and the formula's of Peukert's law and the ideal battery. The numbers for Dualfoil, the diffusion model and Peukert's law have been taken from [19]. 


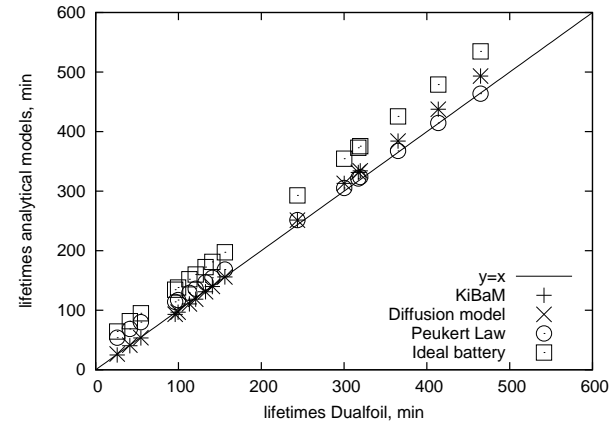

(a) constant load

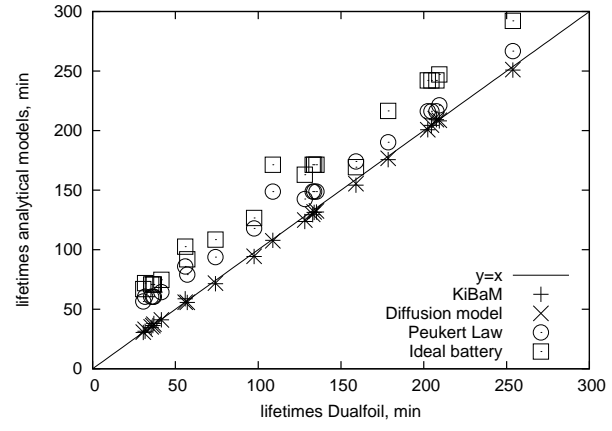

(b) variable load

Figure 10: Computed lifetimes according to the Dualfoil simulation program versus the diffusion model and the KiBaM for constant loads (a) and variable loads (b). Next to the two analytical models, the lifetimes according to the formulas of the ideal battery (1) and Peukert's law (3) are shown.

The lifetimes computed using the KiBaM and diffusion model match very well. The results for continuous discharge only deviate at high discharge currents, as expected from the analysis of the equations, but the difference still is less than $7 \%$. Also, for the variable loads the difference is largest for short battery lifetimes, with a maximum of $5.4 \%$ for Case C21.

Figure 10 shows a plot of the lifetimes computed with both models versus the lifetimes computed with the electro-chemical simulation program Dualfoil. In comparison with Dualfoil both models overestimate the battery lifetime for the low continuous loads (long lifetimes), with errors growing upto $10 \%$. The results of the variable loads are even better, with a maximum error of $5 \%$.

Besides the results of the two models, also the lifetimes according to Peukert's law and the ideal battery model are given. The ideal battery model always predicts longer lifetimes, up to twice as long for high loads, since it does not take into account any loss of capacity due to the rate capacity effect. Also, Peukert's law overestimates the battery lifetimes for most cases. Only for the low continuous loads it gives better predictions than the KiBaM and diffusion model.

\section{$5 \quad$ Limitations of analytical battery models}

In the previous section we have seen that both models give nearly the same results. In this section, all further results are obtained with the KiBaM, but the conclusions also apply to the diffusion model.

With the KiBaM the effect of a varying load on the charge delivered by the battery was analysed in more detail. A square wave, switching between on (1 A) and off (0 A), was used as load. In Figure 11 the charge delivered is shown as a function of the frequency of the periodic load. For low frequencies the delivered charge is constant, because the battery is emptied during the first on-period. Therefore, the charge delivered is equal to the case of continuous discharge at $1 \mathrm{~A}$. When the frequency is increased, one sees a sudden discontinuous increase of the charge delivered by the battery. At the point of this jump, the battery is nearly empty at the end of the first on-period, and it has an off-period to recover some of its capacity. The recovered charge can be used in the next on-period, resulting in a considerable increase 


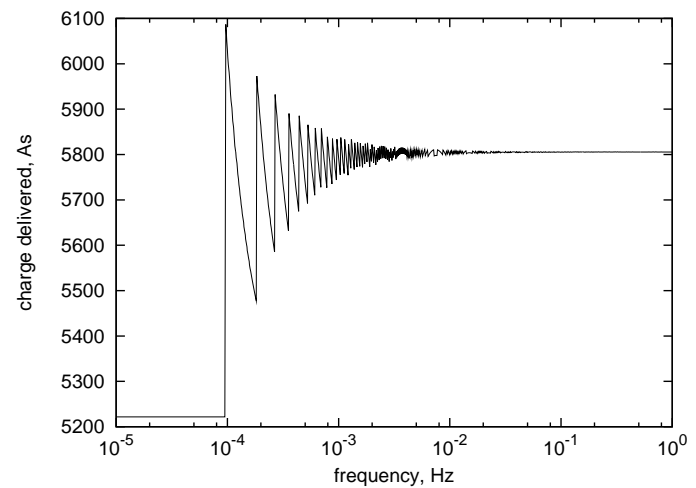

Figure 11: Charge delivered by the battery as a function of the frequency a square wave load. The charge delivered is computed using the KiBaM, with the parameters $c=0.625$, $k=4.5 \cdot 10^{-5} \mathrm{~min}^{-1}$ and the capacity of $7200 \mathrm{As}$.

of the delivered charge. After this increase, the delivered charge slowly decreases when the frequency is further increased. The explanation of this decrease is twofold. First, the offperiod is shorter and therefore there is less time for recovery. Second, the first on-period is shorter and less charge is delivered to the load during this time.

Further increase of the frequency results in a discontinuous increase of the charge delivered each time the battery can recover during an extra off-period, followed again by a slow decrease. The increase gets smaller for higher frequencies since the extra recovery-time decreases. When the frequency is larger than $10^{-2} \mathrm{~Hz}$, the charge delivered is constant again. This is due to the short extra off-time, and the low frequency response at these high frequencies (cf. Section $4.2)$.

For the chosen load and set of battery parameters the charge delivered is highest for a frequency around $10^{-4} \mathrm{~Hz}$. However, the position of the peaks depends highly on the battery parameters and the level of the on-current, and a slight variation might result in a big change in the charge delivered by the battery. In practice, the battery parameters vary even between batteries of the same size and type. Therefore, it does not make sense to do battery lifetime predictions using single traces of a load profile. The used trace could result in a high performance of the battery with one set of the parameters, and a low performance with a slightly different set of parameters.

\section{Conclusions \& Outlook}

Of the battery models available in literature, the analytic models are best suited to be used in performance modelling. The analysis of the KiBaM and diffusion model shows that the $\mathrm{KiBaM}$ is actually a first-order approximation of the diffusion model. The parameters of the KiBaM can be adapted to make a better approximation of the diffusion model. The performed experiments with both models show that this approximation is very good for most practical loads. Therefore, it is better to use the more simple KiBaM model. However, one has to be careful using this type of model when drawing conclusions from only a few workload traces. A slight change in the battery parameters can change the battery lifetime dramatically especially when the load switching frequencies are low. A good way to avoid this problem is 
to make use of stochastic workload models. With these models one can capture the full range of different possible workload traces. This results in a battery lifetime distribution, which tells us the probability of the battery being empty at time $t$ given the type of workload. Comparing these probabilities one can find the best way to use the battery. Slight changes in the battery parameters, now, will not affect the results dramatically. One approach to do this is by using Markov reward models, as described in [2]. Another approach is using priced timed automata [21] to describe the workload, and incorporate the Kinetic Battery Model into this model. This approach can help in finding the best scheduling scheme in a multi-battery system, as shown in first as shown in first case studies in [22]

\section{References}

[1] IEEE Computer, vol. 38, no. 11. IEEE Press, 2005.

[2] L. Cloth, B. R. Haverkort, and M. R. Jongerden, "Computing battery lifetime distributions," in Proceedings of the 37th Annual IEEE/IFIP International Conference on Dependable Systems and Networks (DSN 2007). IEEE Computer Society Press, 2007, pp. $780-789$.

[3] M. R. Jongerden and B. R. Haverkort, "Battery modeling," Centre for Telematics and Information Technology, University of Twente, Technical Report TR-CTIT-08-01, 2008. [Online]. Available: http://eprints.eemcs.utwente.nl/11645/

[4] M. Doyle, T. F. Fuller, and J. Newman, "Modeling of galvanostatic charge and discharge of the lithium/polymer/insertion cell," Journal of the Electrochemical Society, vol. 140, no. 6 , pp. $1526-1533,1993$.

[5] T. F. Fuller, M. Doyle, and J. Newman, "Simulation and optimization of the dual lithium ion insertion cell," Journal of the Electrochemical Society, vol. 141, no. 1, pp. 1 - 10, 1994.

[6] _ - "Relaxation phenomena in lithium-ion-insertion cells," Journal of the Electrochemical Society, vol. 141, no. 4, pp. 982 - 990, 1994.

[7] S. C. Hageman, "Simple PSpice models let you simulate common battery types," Electronic Design News, vol. 38, pp. 117 - 129, 1993.

[8] J. Manwell and J. McGowan, "Lead acid battery storage model for hybrid energy systems," Solar Energy, vol. 50, pp. 399-405, 1993.

[9] — - "Extension of the kinetic battery model for wind/hybrid power systems," in Proceedings of the 5th European Wind Energy Association Conference (EWEC '94), 1994, pp. 284-289.

[10] J. Manwell, J. McGowan, E. Baring-Gould, S. W., and A. Leotta, "Evaluation of battery models for wind/hybrid power system simulation," in Proceedings of the 5th European Wind Energy Association Conference (EWEC '94), 1994, pp. 1182-1187.

[11] D. Rakhmatov and S. Vrudhula, "An analytical high-level battery model for use in energy management of portable electronic systems," in Proceedings of the International Conference on Computer Aided Design (ICCAD'01), 2001, pp. 488-493. 
[12] (2009, July) Fortran programs for the simulation of electrochemical systems. [Online]. Available: http://www.cchem.berkeley.edu/jsngrp/fortran.html

[13] S. Gold, "A PSPICE macromodel for lithium-ion batteries," in 12th Annual Battery Conference on Applications and Advances, 1997, pp. 215-222.

[14] C. Chiasserini and R. Rao, "Pulsed battery discharge in communication devices," in Proceedings of the 5th International Conference on Mobile Computing and Networking, 1999, pp. $88-95$.

[15] — - "A model for battery pulsed discharge with recovery effect," in Wireless Communications and Networking Conference. IEEE Press, 1999, pp. 636 - 639.

[16] —_ "Improving battery performance by using traffic shaping techniques," IEEE Journal on Selected Areas in Communications, vol. 19, no. 7, pp. 1385 - 1394, 2001.

[17] — - "Energy efficient battery management," IEEE Journal on Selected Areas in Communications, vol. 19, no. 7, pp. 1235 - 1245, 2001.

[18] D. Rakhmatov, S. Vrudhula, and D. A. Wallach, "Battery lifetime predictions for energyaware computing," in Proceedings of the 2002 International Symposium on Low Power Electronics and Design (ISLPED '02), 2002, pp. 154-159.

[19] — - "A model for battery lifetime analysis for organizing applications on a pocket computer," IEEE Transactions on VLSI Systems, vol. 11, no. 6, pp. 1019-1030, 2003.

[20] R. Rao, S. B. K. Vrudhula, and N. Chang, "Battery optimization vs energy optimization: which to choose and when?" in Proceedings of the International Conference on Computer Aided Design (ICCAD'05). IEEE Computer Society, 2005, pp. 439-445.

[21] G. Behrmann, K. G. Larsen, and J. I. Rasmussen, "Optimal scheduling using priced timed automata," ACM SIGMETRICS Performance Evaluation Review, vol. 32, no. 4, pp. 34-40, 2005.

[22] M. Jongerden, B. Haverkort, H. Bohnenkamp, and J.-P. Katoen, "Maximizing system lifetime by battery scheduling," in Proceedings of the 39th Annual IEEE/IFIP International Conference on Dependable Systems and Networks (DSN 2009). IEEE Computer Society Press, 2009, pp. 63-72.

\section{A Appendix}




\begin{tabular}{|c|c|c|}
\hline Case & Description & Timing (min) \\
\hline $\mathrm{C} 1$ & IAT-off-IAT & $(0,19.5,26.0)$ \\
\hline $\mathrm{C} 2$ & IAR-off-IAR & $(0,31.0,41.3)$ \\
\hline $\mathrm{C} 3$ & IST-off-IST & $(0,41.0,54.6)$ \\
\hline $\mathrm{C} 4$ & ISR-off-ISR & $(0,74.6,99.5)$ \\
\hline C5 & MPEG-off-MPEG & $(0,105.7,140.9)$ \\
\hline C6 & IAT-off-IAT & $(0,19.5,29.9)$ \\
\hline $\mathrm{C} 7$ & IAT-off-IAT & $(0,19.5,22.1)$ \\
\hline $\mathrm{C} 8$ & IAT-off-IAT & $(0,23.4,29.9)$ \\
\hline C9 & IAT-off-IAT & $(0,15.6,22.1)$ \\
\hline $\mathrm{C} 10$ & Boot-IAT-IAR-MSD-DSD-TSD-WSD-IAD & $(0,0.5,5.5,10.5,35.5,60.5,85.5,110.5)$ \\
\hline $\mathrm{C} 11$ & Boot-WSD-TSD-DSD-MSD-IAR-IAT-IAD & $(0,0.5,25.5,50.5,75.5,100.5,105.5,110.5)$ \\
\hline $\mathrm{C} 12$ & $\begin{array}{l}\text { Boot-WSD-TSD-DSD-MSD-IAR-off-... } \\
\text { Boot-IAT-IAD }\end{array}$ & $\begin{array}{l}(0,0.5,25.5,50.5,75.5,100.5,105.5, \ldots \\
130.5,131.0,136.0)\end{array}$ \\
\hline $\mathrm{C} 13$ & Boot-[IAT-IAR-MSD-DSD-TSD-WSD] ${ }^{5}$-IAD & $\left(0,[0.5,1.5,2.5,7.5,12.5,17.5]_{22.5}^{5}, 110.5\right)$ \\
\hline C14 & Boot-[WSD-TSD-DSD-MSD-IAR-IAT] ${ }^{5}$-IAD & $\left(0,[0.5,5.5,10.5,15.5,20.5,21.5]_{22.5}^{5}, 110.5\right)$ \\
\hline $\mathrm{C} 15$ & MPEG-Dictation-Talk1-WaV1-MPEG & $(0,50.0,100.0,150.0,200.0)$ \\
\hline C16 & WAV1-Talk1-Dictation-MPEG-MPEG & $(0,50.0,100.0,150.0,200.0)$ \\
\hline C17 & WAV1-Talk1-Dictation-off-MPEG-MPEG & $(0,50.0,100.0,150.0,200.0,250.0)$ \\
\hline $\mathrm{C} 18$ & [WAV1-Talk1-Dictation-MPEG] ${ }^{10}$-MPEG & $\left([0,5.0,10.0,15.0]_{20.0}^{10}, 200\right)$ \\
\hline C19 & [WAV2-Talk3-Dictation-MPEG] ${ }^{10}$-MPEG & $\left([0,5.0,10.0,15.0]_{20.0}^{10}, 200\right)$ \\
\hline $\mathrm{C} 20$ & {$[\text { IAR-IAT }]^{\infty}$} & $\left([0,1.0]_{2.0}^{\infty}\right)$ \\
\hline $\mathrm{C} 21$ & {$[\text { IAR-IAT-ISD }]^{\infty}$} & $\left([0,1.0,2.0]_{3.0}^{\infty}\right)$ \\
\hline $\mathrm{C} 22$ & $5.0+(5.0$ per $\min )$ & $(0,1.0,2.0, \ldots)$ \\
\hline
\end{tabular}

Table 3: The simulated variable-load profiles [19] 